\title{
Predictors of Death in Trauma Patients who are Alive on Arrival at Hospital
}

\author{
Rob A. Lichtveld', Ivo F. Panhuizen², Ronald B. J. Smit ${ }^{3}$, Hermann R. Holtslag ${ }^{4}$, \\ Christian van der Werken ${ }^{5}$
}

\begin{abstract}
Objective: To determine which factors predict death occurring in trauma patients who are alive on arrival at hospital Design Prospective cohort study Method Data were collected from 507 trauma patients with multiple injuries, with a Hospital Trauma Index-Injury Severity Score of 16 or more, who were initially delivered by the Emergency Medical Services to the Emergency Department of the University Medical Centre Utrecht (UMCU) during the period 1999-2000.

Results: Univariate analysis showed that every year of age increase resulted in a $2 \%$ greater risk of death. If the patient had been intubated at the scene of the accident, this risk was increased 4.3-fold. Every point of increase in the Triage Revised Trauma Score (T-RTS) reduced the risk of death by $30 \%$. A similar (but inverse) tendency was found for the HTI-ISS score, with every point of increase resulting in a $5 \%$ greater risk of death. There was a clear relationship between the base excess ( $\mathrm{BE}$ ) and hemoglobin $(\mathrm{Hb})$ levels and the risk of death, the latter being increased by $8 \%$ for each $\mathrm{mmol} / \mathrm{l}$ drop in BE, and reduced by $22 \%$ for each $\mathrm{mmol} / \mathrm{l}$ increase in $\mathrm{Hb}$. The risk of death occurring was 2.6 times higher in cases with isolated neurotrauma. These associations hardly changed in the multivariate analysis; only the relation with having been intubated at the scene disappeared.

Conclusion: The risk of severely injured accident patients dying after arriving in hospital is mainly determined by the T-RTS, age, presence of isolated neurolog-
\end{abstract}

ical damage, $\mathrm{BE}$ and $\mathrm{Hb}$ level. Skull/brain damage and hemorrhage appear to be the most important causes of death in the first $24 \mathrm{~h}$ after the accident.

The time interval between the accident and arrival at the hospital does not appear to affect the risk of death.

\section{Key Words}

Scoring systems $\cdot$ Polytrauma $\cdot$ Traumatic brain injury - Thorax and abdominal trauma - Trauma registries - Polytrauma managment including prehospital and shockroom - Multiple trauma

Eur J Trauma Emerg Surg 2007;33:46-51

DOI 10.1007/s00068-007-6097-6

\section{Introduction}

In the Netherlands, some 5,000 people a year die of unnatural causes; in $60 \%$ of the cases, death occurs in a non-hospital setting. Death is considered to be unnatural if caused by external factors, and natural if caused by old age or following an illness. Most non-natural deaths are the result of accidents in the home, followed by suicide and traffic accidents.

Almost half of all fatal accident victims die immediately at the scene of the accident, due to injury to the brain or heart or because large blood vessel rupture. About one-third of them die a few hours later, again as a result of brain damage or internal bleeding. Death occurring at a later stage, varying from a few

\footnotetext{
${ }^{1}$ RAVU - EMS, Nassaustraat 127, Maarssen, Utrecht, $3601 \mathrm{BE}$ The Netherlands,

${ }^{2}$ Department of Anaesthesiology, University Medical Centre St Radboud, Nijmegen, The Netherlands,

${ }^{3}$ Forensic Medicine, Municipal Health Authority, Amsterdam, The Netherlands,

${ }^{4}$ Rehabilitation Medicine, University Medical Centre St Radboud, Nijmegen, The Netherlands,

${ }^{5}$ Department of Surgery, Universtiy Medical Center Utrecht, Utrecht, The Netherlands.
}

Received: March 7, 2006; revision accepted: November 4, 2006; Published Online: February 27, 2007 
days to weeks or even months after an accident, is usually caused by late complications resulting from the accident and often no longer bears directly on the original injury [1].

In severely injured trauma patients, care in the acute phase focuses particularly on ensuring that the time spent at the scene of the accident is as short as possible, as well as on rapid and adequate oxygenation and improving organ perfusion to increase the chances of survival. On the basis of data from the Vietnam war, the generally accepted policy is that severely injured trauma patients must undergo definitive treatment in hospital within $1 \mathrm{~h}$ of the injury (the golden hour) to optimize the chances of survival [2]. Although some information is available about the period prior to hospital treatment, not enough is known about the predictors of death when patients actually arrive in hospital alive.

If tissue perfusion is insufficient, the body switches to anaerobic metabolism [3, 4]. In this form of metabolism, lactate is released into the circulation, leading to acidosis and a decrease in base excess. Base excess is defined as the amount of acid required to titrate 11 of blood to normal $\mathrm{pH}$ (7.40) under normal conditions of $\mathrm{p}_{\mathrm{a}} \mathrm{O}_{2}, \mathrm{p}_{\mathrm{a}} \mathrm{CO}_{2}$ and a temperature of $37.0^{\circ} \mathrm{C}$. The range of normal values for base excess is $\pm 2.5 \mathrm{mmol} / \mathrm{l}$. Davis et al. [5] showed that a base excess below $6 \mathrm{mmol} / 1$ is closely associated with the severity of the injuries and the risk of death.

The uncertainty with regard to the predictors of death led us to address the following question in this study: what factors predict the risk that trauma patients who are alive on arrival at hospital will die?

\section{Patients and Methods}

The University Medical Centre Utrecht is one of the ten trauma centers in the Netherlands. Its trauma catchment area consists of an urbanized region in the center of the country, with a total population of 1.1 million (density: 813 inhabitants per square kilometer). If at all possible, severely injured patients in the Utrecht region are transported to this hospital.

During the period from January 1999 to December 2000, all severely injured patients with a Hospital Trauma Index-Injury Severity Score (HTI-ISS) [6] of at least 16 who were initially delivered by the ambulance service to the Emergency Department of the UMCU were included in a prospective cohort study.

The HTI-ISS is a combined anatomical and physiological scale, developed to determine the overall severity of a patient's injuries. The HTI reflects the severity of the injuries to six parts of the body in a score of 1 (minimal) to 5 (life-threatening). The ISS is calculated from the sum of the square of each of the three highest HTI units (ISS) [6]. For example, a patient with an impression fracture of the skull (3 points), loss of $500 \mathrm{ml}$ blood ( 2 points) and a closed humerus fracture ( 2 points) has an HTI-ISS of $17\left(3^{2}+2^{2}+2^{2}\right)$.

The ambulance nurse and/or Mobile Medical Team (MMT) doctor kept records of all patient contacts, including data such as

- personal details of the patient;

- timing of important events (time of the accident, time when the ambulance/MMT reached the patient, time of ambulance/MMT leaving with the patient to the hospital and time of arrival of the patient at the emergency department);

- the Triage Revised Trauma Score (T-RTS);

- the diagnosis established at the scene of the accident;

- treatment applied in the pre-hospital setting (Table 1).

The T-RTS [7], which makes use of physiological parameters like breathing rate, blood pressure and the level of consciousness measured with the Glasgow Coma Scale (GCS) [8] is used in ambulance care to classify trauma patients according to the severity of the injury (see Table 2, T-RTS). The T-RTS varies between 0 (no sign of life) and 12 (normal vital functions). We used the T-RTS on the scene in the analysis. An arterial blood sample was taken on arrival at hospital to determine $\mathrm{Hb}, \mathrm{Ht}$, base excess, bicarbonate, $\mathrm{p}_{\mathrm{a}} \mathrm{O}_{2}, \mathrm{p}_{\mathrm{a}} \mathrm{CO}_{2}$ and $\mathrm{SaO}_{2}$. We tested the value of $\mathrm{Hb}$ and base excess as potential predictors. When the patient was discharged, his/her data were documented in a letter to their family doctor (GP). Data used in the present study included the discharge diagnoses, the nature of the injuries and the cause of death if the patient died. Finally, any deaths resulting from the trauma were recorded during an 18-month follow-up period. The guidelines and protocols for ambulance and emergency services did not change during the study period.

\section{Statistical Analysis}

The Chi-square and $t$ tests or the Mann-Whitney $U$ test were used to assess differences between the various groups. Partial correlation was used to determine the influence of the severity of the injury and the passage of time on the level of base excess. Univariate and multivariate Cox regression analyses were used to identify the factors that influenced the risk of the pa- 
Table 1. Baseline characteristics.

\begin{tabular}{llll}
\hline Variables & Total $(\mathbf{n}=\mathbf{5 0 7})$ & Survivors $(\mathbf{n}=\mathbf{4 0 7})$ & Non-survivors $(\mathbf{n}=\mathbf{1 0 0})$ \\
\hline Age (years) & $36.8 \pm 19.6$ & $34.9 \pm 18.1$ & $44.4 \pm 23.4$ \\
Gender (\% male) & $367(72)$ & $293(72)$ & $74(74)$ \\
Intubation (\%) & $75(15)$ & $36(9)$ & $39(39)$ \\
Intravenous drip (\%) & $424(87)$ & $337(87)$ & $87(89)$ \\
T-RTS & $10.3 \pm 2.5$ & $10.9 \pm 1.7$ & $8.0 \pm 3.5$ \\
HTI-ISS & $26.5 \pm 13.0$ & $24.5 \pm 10.2$ & $34.8 \pm 18.6$ \\
Average total pre-hospital time (min) & $61.9 \pm 26.8$ & $61.9 \pm 24.7$ & $62.1 \pm 33.4$ \\
Average time at scene of accident (min) & $29.4 \pm 16.2$ & $29.6 \pm 16.2$ & $28.6 \pm 16.1$ \\
Transport time (min) & $17.8 \pm 11.0$ & $18.2 \pm 10.5$ & $16.5 \pm 12.5$ \\
Base excess (mmol/l; median: minimum, maximum) & $-3.3 ;-26.1-6.12$ & $-3.1 ;-22.3-6.12$ & $-4.2 ;-26.1-2.4$ \\
Hb (mmol/l) & $7.6 \pm 1.4$ & $7.7 \pm 1.3$ & $7.1 \pm 1.6$
\end{tabular}

Values are means $\pm S D$, unless otherwise stated

tient dying. The level of significance $\alpha$ was set at 0.05 , unless otherwise stated. All analyses were carried out using SPSS version 12.

\section{Results}

From January 1999 to December 2000, 507 severely injured patients (367 (72.4\%) male and 140 female; 493 $(97.2 \%)$ blunt trauma) met the inclusion criteria. The characteristics of the study group are summarized in Table 2.

The average age of the men was 35.8 years $(\mathrm{SD}=18.4)$ and that of the women $39.5(\mathrm{SD}=22.4)$. In total, 100 patients $(19.7 \%)$ died, 92 during admission and 8 during the 18-month follow-up period. The group of patients with a T-RTS of 0 or 1 (all having suffered blunt trauma, $\mathrm{n}=10$ ) had no survivors. The mechanism of injury was high-energy trauma in $84 \%$, and the causes of all injuries were traffic accidents in $60 \%$ of cases and falls from staircases or heights in 30\%.

The average time spent at the scene of the accident was about $30 \mathrm{~min}$, for those who died as well as those who survived. Cox regression was used to assess a possible correlation between the risk of dying and the duration of the pre-hospital phase, especially in patients with injuries to the head, chest and abdomen and to the ribs, clavicle and shoulder, back, pelvis, hip and limbs. For none of these injuries did there appear to be a link between the duration of the pre-hospital phase and the risk of dying $(\mathrm{p}>0.05)$.

Some $45.2 \%$ of patients with an initial (on-scene) T-RTS lower than 10 had a base excess of less than -6 , whereas for those with an initial T-RTS of 10,11 or 12 , the base excess was -6 in only $19.2,16.4$ and $19.2 \%$ of the patients, respectively $(\mathrm{p}<0.001)$. Among patients with an HTI-ISS less than 34 , a base excess of less than -6 was found in $38.4 \%$, compared to $61.6 \%$ of the patients whose HTI-ISS was higher than 34 $(\mathrm{p}<0.001)$.

There was no relation between the level of base excess and the time interval between the accident and the arrival at the UMCU, corrected for T-RTS and HTI-ISS $(\mathrm{p}=0.618)$.

Table 2 shows the relationships between a number of patient characteristics and variables measured at baseline and the risk of death occurring within

Table 2. Cox regression analysis of patient characteristics and baseline measurements as determinants of death within 1.5 years.

\begin{tabular}{|c|c|c|}
\hline & \multicolumn{2}{|c|}{ Hazard ratios $(95 \% \mathrm{CI})$} \\
\hline & Univariate & Multivariate \\
\hline Age (years) & $1.02(1.01-1.03)^{*}$ & $1.02(1.01-1.03)^{*}$ \\
\hline Gender (male) & $0.6(0.7-1.7)$ & \\
\hline Intubation (yes/no) & $4.5(3.0-6.7)^{*}$ & $1.1(0.7-1.9)$ \\
\hline Intravenous drip (yes/no) & $1.2(0.6-2.2)$ & \\
\hline T-RTS & $0.7(0.7-0.8)^{*}$ & $0.8\left(0.7-0.9^{*}\right.$ \\
\hline HTI-ISS & $1.1(1.0-1.1)^{*}$ & $1.02(1.01-1.04)^{* *}$ \\
\hline $\begin{array}{l}\text { Time between accident and } \\
\text { arrival at hospital (min) }\end{array}$ & $1(0.99-1.01)$ & \\
\hline $\begin{array}{l}\text { Time at the scene of } \\
\text { the accident ( } \min )\end{array}$ & $0.99(0.98-1.01)$ & \\
\hline Transport time to hospital (min) & $0.98(0.96-1.01)$ & \\
\hline Base excess (mmol/l) & $0.92(0.89-0.95)^{*}$ & $0.95(0.91-0.99)^{* *}$ \\
\hline $\mathrm{Hb}$ (mmol/l) & $0.78(0.69-0.88)^{*}$ & $0.89(0.77-0.99)$ \\
\hline \multicolumn{3}{|l|}{ Neurotrauma } \\
\hline None & Reference & Reference \\
\hline Combined with other injuries & $1.6(1.0-2.7)^{* *}$ & $1.5(0.9-2.4)$ \\
\hline Isolated & $3.4(2.1-5.8) *$ & $4.3(2.4-7.7)^{*}$ \\
\hline
\end{tabular}

95\% CI: $95 \%$ confidence interval

${ }^{*} p<0.001,{ }^{* *} p<0.05$. The significant variables in the second (Univariate) column have been subjected to multivariate analysis 
1.5 years after the accident. Univariate analysis showed that there was a $2 \%$ greater risk of death for each year of age increase. Elderly patients of 55 years of age and over had a 2.2-fold (CI 1.5-3.4; p < 0.001) higher risk of dying than patients below 55 years of age.

If the patient had been intubated at the scene of the accident, the risk of dying was increased 4.5-fold.

For each point by which the T-RTS score increased, the risk of dying dropped by $30 \%$. A similar but inverse tendency was seen for the HTI-ISS score. A 1-point increase in the HTI-ISS led to a $5 \%$ greater risk of death. The base excess (BE) and hemoglobin $(\mathrm{Hb})$ levels were clearly related to the risk of dying: each $\mathrm{mmol} / \mathrm{l}$ drop in $\mathrm{BE}$ increased the risk of death by $8 \%$, while each $\mathrm{mmol} / \mathrm{l}$ decrease in $\mathrm{Hb}$ increased this risk by $22 \%$. The risk that the patient would die was 3.4 times higher in cases with isolated neurotrauma. These results hardly changed in the multivariate analysis: only the associations with having been intubated at the scene and $\mathrm{Hb}$ levels disappeared.

Slightly more than half of all deaths $(51.9 \%)$ occurred in the first $24 \mathrm{~h}$ after the accident, and $80.2 \%$ in the first week. Twenty-two patients died in the emergency department, twelve of whom had been resuscitated on arrival at the UMCU or required immediate resuscitation. The causes of death of the patients who died in the emergency department were hemorrhage (eight patients), severe head injuries (ten patients), high level lesion of the spinal cord (one patient) and compression injury to the chest and abdomen (one patient). The causes of death of all patients who passed away within $24 \mathrm{~h}(\mathrm{n}=42)$ were severe head injuries (23 patients, $54.8 \%$ ), hemorrhage (12 patients; $28.6 \%$ ), heart rhythm disturbance (4 patients; $9.5 \%$ ), respiratory insufficiency (2 patients; $4.8 \%$ ) and high-level spinal injury (1 patient, $2.4 \%$ ).

Patients who died as a result of severe head injuries had an average base excess of -5.36 , versus -12.93 for patients who died as a consequence of hemorrhage $(\mathrm{p}=0.008)$.

\section{Discussion}

The aim of this study was to identify predictors of the risk of death in severely injured patients.

The total mortality of the study population with an HTI-ISS of 16 or more was $19.7 \%$. About half of all deaths occurred in the first $24 \mathrm{~h}$ after the accident. In these patients, skull/brain injury was the most important cause of death (more than half), followed by hemorrhage (about one-third of cases).
The age of patients played a crucial role. This relation has been abundantly reported in the literature and is associated with the greater risk of complications, decrease in physical reserves and more existing comorbidity in older patients [9]. Particularly elderly patients (defined as 55 years or over) had an increased risk of dying.

The risk of death was strongly dependent on the severity of injury, in terms of both T-RTS and HTIISS. It decreased by $30 \%$ with every point of increase in T-RTS score.

The time interval between the accident and the patient's arrival at the hospital did not differ between survivors and deceased persons, and had no influence on the risk of the patient dying. The time spent at the scene of the accident - about $30 \mathrm{~min}$ on average - was rather long, considering the fact that the target time mentioned in the Dutch National Ambulance Service Protocol is $10 \mathrm{~min}$ for unstable patients [10]. The greatly increased risk of dying for patients who had been intubated at the scene could largely be explained by the severity of the injury but was still slightly increased after correction for age, T-RTS score, BE, $\mathrm{Hb}$ and neurotrauma. Models to predict death have been proposed by several research teams. Guzzo et al. demonstrated that their model had a sensitivity of $45 \%$ and a specificity of $96 \%$, using the variables GCS, base excess, need for transfusion, ISS and age [11]. Other research teams demonstrated that negative base excess clearly reflects injury severity and predict mortality [12-21].

Of the patients who were resuscitated prior to arrival at the hospital or who required resuscitation on arrival at the emergency department, none survived their injuries. None of the patients with a T-RTS of 0 or 1 (all with blunt trauma) survived. This could be a reason to desist from resuscitating such patients in accidents where there are more injured parties and where the capacity for treatment is limited.

Stockinger et al. [22] found no survivors of penetrating trauma and fewer than $1 \%$ for overall trauma with a T-RTS score of 0 . Their findings support the guidelines for pre-hospital cessation of resuscitative efforts published by the National Association of Emergency Physicians (NAEP) and the American College of Surgeons Committee on Trauma. According to these guidelines, victims of penetrating trauma with a pre-hospital RTS of 0 (i.e., a combination of no respiratory rate, no systolic blood pressure and a Glasgow Coma Score of 3) should be regarded as "dead at the scene" [23]. By contrast, Pickens et al. [24] stated that these NAEP guidelines should not be 
adopted until more thorough studies had been conducted. In their retrospective cohort study, $7.6 \%$ of the patients who met the criteria for non-treatment nevertheless survived to discharge.

More research is required to determine for which categories of accident victims resuscitation is meaningful.

In our study, a longer time interval between the accident and arrival at the hospital did not lead to a greater risk of death for any of the injuries investigated. The time factor would appear to be subordinate in terms of pre-hospital treatment of severe trauma patients. This finding is in accordance with Lerner's study, which found that after correction for ISS, patient characteristics and treatment aspects, there was no relation between the time from the moment of the accident to the patient's arrival at hospital on the one hand and the risk of death on the other [25]. The conclusions of Lerner's study and our own should, however, be interpreted with some caution. They do not mean, for instance that the usual rule of ambulance care that only essential treatment should be carried out at the scene and that the patient should then be transported quickly to hospital should be discouraged. Resuscitation at the site of the accident and resuscitation in the emergency room differ vastly and are delivered by teams with different levels of expertise and training. This means that it is necessary to present the severely injured patient to a level 1 trauma center as soon as possible. Further prospective randomized studies will have to assess the best practice for ambulance care.

Our findings show a greater risk of death among trauma patients with poor organ perfusion. The base excess, which decrease when tissue perfusion is inadequate as a result of anaerobic metabolism, was significantly lower in the patients who died. The base excess in patients who died as a result of hemorrhage was significantly lower than in patients who died as a consequence of skull/brain injury. This observation is in accordance with the findings by Kaplan et al. [26], who demonstrated that in cases of severe blood vessel damage, the level of base excess can definitely distinguish the survivors from the non-survivors. The higher the $\mathrm{Hb}$ level on arrival at the emergency department, the lower the risk of the patient dying.

\section{Conclusion}

The risk of death occurring after the arrival of severely injured accident patients in hospital is mainly determined by T-RTS, age, presence of isolated neurological damage, base excess and $\mathrm{Hb}$. Severe head injuries and hemorrhage appear to be the most important causes of death in the first $24 \mathrm{~h}$ after the accident. The time elapsing between the accident and arrival at the hospital does not appear to influence the risk of death.

\section{References}

1. Cales RH, Trunkey DD. Preventable trauma deaths. A review of trauma care systems development. JAMA 1985;254:1059-63.

2. Lerner EB, Moscati RM. The golden hour: scientific fact or medical "urban legend"?. Acad Emerg Med 2001;8:758-6o.

3. Siegel JH, Rivkind Al, Dalal S, Goodarzi S. Early physiologic predictors of injury severity and death in blunt multiple trauma. Arch Surg 1990;125:498-508.

4. Davis JW, Kaups KL, Parks SN. Base deficit is superior to $\mathrm{pH}$ in evaluating clearance of acidosis after traumatic shock. J Trauma 1998;44:114-8.

5. Davis JW, Kaups KL. Base deficit in the elderly: a marker of severe injury and death. J Trauma 1998;45:873-7.

6. Baker SP, O'Neill B, Haddon W Jr, Long WB. The injury severity score: a method for describing patients with multiple injuries and evaluating emergency care. J Trauma 1974;14:187-96.

7. Champion HR, Sacco WJ, Copes WS, Gann DS, Gennarelli TA, Flanagan ME. A revision of the Trauma Score. J Trauma 1989;29:623-29.

8. Teasdale G, Jennett B. Assessment of coma and impaired consciousness. A practical scale. Lancet 1974;2:81-4.

9. Perdue PW, Watts DD, Kaufmann CR, Trask AL. Differences in mortality between elderly and younger adult trauma patients: geriatric status increases risk of delayed death. J Trauma 1998;45:805-10.

10. Hartman JAM, Lichtveld RA, de Vries GMJ, Wolde WLM. Landelijk Protocol Ambulancezorg. Stichting LAMP, Zwolle, 2005.

11. Guzzo JL, Bochicchio GV, Napolitano LM, Malone DL, Meyer W, Scalea TM. Prediction of outcomes in trauma: anatomic or physiologic parameters?. J Am Coll Surg 2005;201:891-7.

12. Davis JW, Mackersie RC, Holbrook TL, Hoyt DB. Base deficit as an indicator of significant abdominal injury. Ann Emerg Med 1991;20:842-4.

13. Davis JW, Kaups KL, Parks SN. Effect of alcohol on the utility of base deficit in trauma. J Trauma 1997:43:507-10.

14. Huerta S, Bui T, Porral D, Lush S, Cinat M. Predictors of morbidity and mortality in patients with traumatic duodenal injuries. Am Surg 2005;71:763-7.

15. Kincaid EH, Chang MC, Letton RW, Chen JG, Meredith JW. Admission base deficit in pediatric trauma: a study using the National Trauma Data Bank. J Trauma 2001;51:332-5.

16. Martin MJ, Weng J, Demetriades D, Salim A. Patterns of injury and functional outcome after hanging: analysis of the National Trauma Data Bank. Am J Surg 2005;190:836-40.

17. Nirula R, Gentilello LM. Futility of resuscitation criteria for the "young" old and the "old" old trauma patient: a national trauma data bank analysis. J Trauma 2004;57:37-41.

18. Peterson DL, Schinco MA, Kerwin AJ, Griffen MM, Pieper P, Tepas JJ. Evaluation of initial base deficit as a prognosticator of outcome in the pediatric trauma population. Am Surg 2004;70:326-8.

19. Rutherford EJ, Morris JA Jr, Reed GW, Hall KS. Base deficit stratifies mortality and determines therapy. J Trauma 1992;33:417-23. 
20. Tremblay LN, Feliciano DV, Rozycki GS. Are resuscitation and operation justified in injured patients with extreme base deficits (less than -20)?. Am J Surg 2003;186:597-600.

21. Rixen D, Raum M, Bouillon B, Lefering R, Neugebauer E. Base deficit development and its prognostic significance in posttrauma critical illness: an analysis by the trauma registry of the Deutsche Gesellschaft fur unfallchirurgie. Shock 2001;15:83-9.

22. Stockinger ZT, McSwain NE Jr. Additional evidence in support of withholding or terminating cardiopulmonary resuscitation for trauma patients in the field. J Am Coll Surg 2004;198:227-31.

23. Hopson LR, Hirsh E, Delgado J, Domeier RM, MCSwain NE, Krohmer J. Guidelines for withholding or termination of resuscitation in prehospital traumatic cardiopulmonary arrest: joint position statement of the National Association of EMS Physicians and the American College of Surgeons Committee on Trauma. J Am Coll Surg 2003;196:106-12.

24. Pickens JJ, Copass MK, Bulger EM. Trauma patients receiving CPR: predictors of survival. J Trauma 2005;58:951-8.
25. Lerner EB, Billittier AJ, Dorn JM, Wu YW. Is total out-of-hospital time a significant predictor of trauma patient mortality?. Acad Emerg Med 2003;10:949-54.

26. Kaplan $\sqcup$, Kellum JA. Initial pH, base deficit, lactate, anion gap, strong ion difference, and strong ion gap predict outcome from major vascular injury. Crit Care Med 2004;32:1120-4.

\section{Address for Correspondence}

Rob A. Lichtveld

RAVU - EMS, Nassaustraat 127

Maarssen, Utrecht, $3601 \mathrm{BE}$

The Netherlands

Phone $(+31 / 34) 6242704$, Fax $(+31 / 30) 8501219$

e-mail: LMMC@wxs.nl, r.lichtveld@ravu.nl 